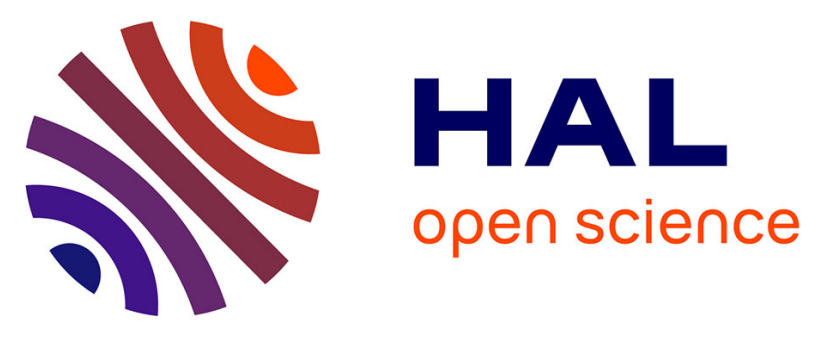

\title{
Decoding biomineralization: Interaction of a Mad10-derived peptide with magnetite thin films
}

Anna Pohl, Florian Berger, Ruby M.A. Sullan, Carmen Valverde-Tercedor, Kinga Freindl, Nika Spiridis, Christopher T. Lefèvre, Nicolas F Menguy, Stefan Klumpp, Kerstin G. Blank, et al.

\section{To cite this version:}

Anna Pohl, Florian Berger, Ruby M.A. Sullan, Carmen Valverde-Tercedor, Kinga Freindl, et al.. Decoding biomineralization: Interaction of a Mad10-derived peptide with magnetite thin films. Nano Letters, 2019, 19, pp.8207 - 8215. 10.1021/acs.nanolett.9b03560 . cea-02308638

\section{HAL Id: cea-02308638 https://hal-cea.archives-ouvertes.fr/cea-02308638}

Submitted on 8 Nov 2020

HAL is a multi-disciplinary open access archive for the deposit and dissemination of scientific research documents, whether they are published or not. The documents may come from teaching and research institutions in France or abroad, or from public or private research centers.
L'archive ouverte pluridisciplinaire HAL, est destinée au dépôt et à la diffusion de documents scientifiques de niveau recherche, publiés ou non, émanant des établissements d'enseignement et de recherche français ou étrangers, des laboratoires publics ou privés.

\section{(c)(1)}

Distributed under a Creative Commons Attribution| 4.0 International License 


\title{
Decoding Biomineralization: Interaction of a Mad10-Derived Peptide with Magnetite Thin Films
}

\author{
Anna Pohl, ${ }^{\dagger, \Delta, \ddagger}$ Florian Berger, ${ }^{\S, \ddagger}$ Ruby M. A. Sullan, ${ }^{\Delta, \ddagger} \diamond$ Carmen Valverde-Tercedor, ${ }^{\dagger}$
}

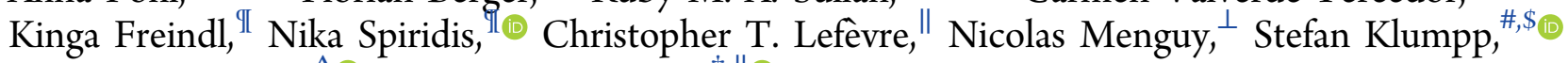
Kerstin G. Blank, ${ }^{, \Delta}{ }_{\odot}$ and Damien Faivre ${ }^{*},+, \|_{\odot}$

${ }^{\dagger}$ Department of Biomaterials, Max Planck Institute of Colloids and Interfaces, Am Mühlenberg 1, 14476 Potsdam, Germany

${ }^{\Delta}$ Mechano(bio)chemistry, Max Planck Institute of Colloids and Interfaces, Am Mühlenberg 1, 14476 Potsdam, Germany

${ }^{\S}$ Laboratory of Sensory Neuroscience, The Rockefeller University, 1230 York Avenue, New York 10065, United States

IJ Jerzy Haber Institute of Catalysis and Surface Chemistry, Polish Academy of Sciences, Niezapominajek 8, 30-239 Krakow, Poland

"Aix-Marseille Université, CEA, CNRS, BIAM, 13108 Saint Paul lez Durance, France

${ }^{\perp}$ Sorbonne Université, UMR CNRS 7590, IRD. MNHN, Institut de Minéralogie, Physique des Matériaux et Cosmochimie IMPMC, 4 Place Jussieu, 75005 Paris, France

\#Department of Theory \& Bio-Systems, Max Planck Institute of Colloids and Interfaces, Am Mühlenberg 1, 14476 Potsdam, Germany

${ }^{\$}$ Institute for the Dynamics of Complex Systems, University of Göttingen, Friedrich Hund Platz 1, 37077 Göttingen, Germany

\section{Supporting Information}

ABSTRACT: Protein-surface interactions play a pivotal role in processes as diverse as biomineralization, biofouling, and the cellular response to medical implants. In biomineralization processes, biomacromolecules control mineral deposition and architecture via complex and often unknown mechanisms. For studying these mechanisms, the formation of magnetite nanoparticles in magnetotactic bacteria has become an excellent model system. Most interestingly, nanoparticle morphologies have been discovered that defy crystallographic rules (e.g., in the species Desulfamplus magnetovallimortis strain BW-1). In certain conditions, this strain mineralizes bullet-shaped magnetite nanoparticles, which exhibit defined (111) crystal faces and are elongated

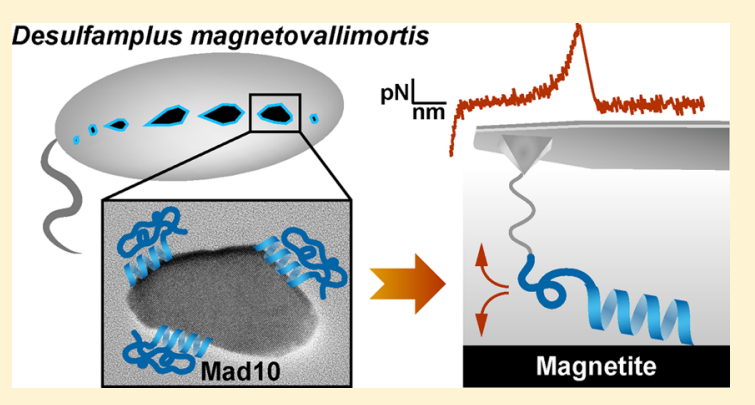
along the $[100]$ direction. We hypothesize that surface-specific protein interactions break the nanoparticle symmetry, inhibiting the growth of certain crystal faces and thereby favoring the growth of others. Screening the genome of BW-1, we identified Mad10 (Magnetosome-associated deep-branching) as a potential magnetite-binding protein. Using atomic force microscope (AFM)-based single-molecule force spectroscopy, we show that a Mad10-derived peptide, which represents the most conserved region of Mad10, binds strongly to (100)- and (111)-oriented single-crystalline magnetite thin films. The peptidemagnetite interaction is thus material- but not crystal-face-specific. It is characterized by broad rupture force distributions that do not depend on the retraction speed of the AFM cantilever. To account for these experimental findings, we introduce a three-state model that incorporates fast rebinding. The model suggests that the peptide-surface interaction is strong in the absence of load, which is a direct result of this fast rebinding process. Overall, our study sheds light on the kinetic nature of peptide-surface interactions and introduces a new magnetitebinding peptide with potential use as a functional coating for magnetite nanoparticles in biotechnological and biomedical applications.

KEYWORDS: magnetite, magnetotactic bacteria, magnetite-binding peptide, single-molecule force spectroscopy, biomineralization, organics-inorganics interaction

$\mathrm{U}$ nderstanding the interaction between biomolecules and inorganic surfaces is of fundamental and applied interest in areas ranging from the control of crystal growth in synthetic and biological systems ${ }^{1,2}$ to the synthesis of biocomposites, ${ }^{3-5}$ biomedical implants, ${ }^{6,7}$ and functional coatings. ${ }^{8}$ Knowledge about the binding mechanisms and kinetics of specific interactions between biomacromolecules and inorganic surfaces is required before these interactions can be utilized in a controlled manner for the in vitro synthesis of tailor-made materials. In this regard, biomineralizing organisms offer a variety of model systems where organic-inorganic interactions can be studied.

Magnetotactic bacteria (MTB), which form magnetite nanoparticles (MNPs), ${ }^{1,2}$ are a highly powerful model for

Received: August 29, 2019

Revised: September 27, 2019

Published: September 30, 2019 
investigating the underlying molecular interactions and mechanisms. In MTB, proteins are known to control the size and organization of MNPs in specific organelles, termed magnetosomes. MNP properties are species-dependent ${ }^{9,10}$ and can be altered using genetic modifications. ${ }^{2,11}$ Most interestingly, MTB form elongated particles of a mineral (magnetite) that is known to crystallize in the space group $F d \overline{3} m$. The inverse spinel structure of magnetite is a cubic face-centered crystal structure with two sublattices where usually only isometric (cuboctahedric or rounded) particles are expected. It has been hypothesized that specific proteins are responsible for this crystallography-defying morphology and that these proteins specifically interact with certain crystal faces. ${ }^{10,12,13}$ Only a very limited number of magnetite-binding proteins have been investigated so far. Mms6 was the first protein to be studied both in vitro ${ }^{12,14-16}$ and in vivo ${ }^{17}$ and is now considered to mainly impact the size of the particles. ${ }^{17}$ Despite numerous efforts, no definitive morphology-determining proteins have been identified. As the (magnetic) properties of MNPs are morphology-dependent, ${ }^{18,19}$ control over particle morphology will directly impact potential applications of MNPs. ${ }^{20}$

Here, we focus on Desulfamplus magnetovallimortis strain BW-1. In certain conditions, this strain mineralizes elongated, bullet-shaped MNPs, which defy crystallographic rules. ${ }^{21,22}$ We first investigate the morphology of these elongated MNPs and perform a bioinformatics analysis of the BW-1 genome to identify proteins that are putatively involved in magnetite biomineralization and, in particular, MNP morphology control. We hypothesize that these proteins may bind to specific crystal faces of the mineral, thereby promoting crystal growth along specific crystal directions while blocking others. To characterize the protein-magnetite interaction, we use atomic force microscopy (AFM)-based single-molecule force spectroscopy (SMFS). SMFS is a powerful method for studying proteinsurface interactions, ${ }^{23-29}$ as it allows for measuring binding and unbinding events on nontransparent, crystalline materials. Most importantly, it allows for investigating crystal-face-specificity, probing the possible interaction with magnetite (100)- and (111)-oriented single-crystalline thin films. Focusing on the putative magnetite-binding protein Mad10 (Magnetosomeassociated deep-branching 10), we show that this previously uncharacterized protein displays strong binding to magnetite even though the interaction does not appear to be crystal-facespecific.

Identification of the Putative Magnetite-Binding Protein Mad10. Whereas most MTB synthesize almost spherical MNPs, ${ }^{2}$ the MNPs observed in Desulfamplus magnetovallimortis strain BW-1 exhibit a less-studied bullet-shaped morphology. ${ }^{9,21}$ We therefore started with a characterization of the MNP morphology (Figure 1), using high-resolution transmission electron microscopy. Strikingly, well-defined $\{111\}$ surfaces are observed at the base of the particle, while the particle elongates along the [100] direction. The elongated faces are characterized by higher Miller indices, which led us to speculate that biomolecules may recognize and stop the growth of the $\{111\}$ faces as previously suggested by Li et al. ${ }^{13}$

Using input from bioinformatics, it has been proposed that some Mad proteins might be involved in the morphological control of these elongated MNPs in BW- $1 .{ }^{22}$ We therefore focus on these Mad proteins, expressed by the mad gene cluster. ${ }^{22}$ Thirty mad genes are present in BW-1, and 11 of these genes (mad1 - mad11) are putatively involved in the synthesis of magnetite. ${ }^{22}$ The corresponding amino acid sequences were
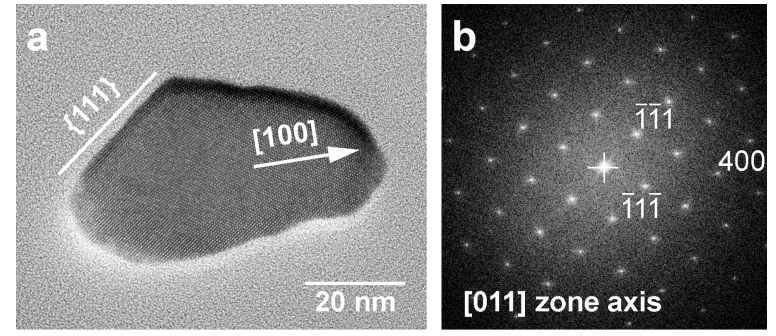

Figure 1. High-resolution transmission electron microscopy of one characterisitic bullet-shaped magnetite nanoparticle found in Desulfamplus magnetovallimortis strain BW-1. (a) Micrograph of the nanoparticle. (b) From the FFT indexation, it appears that the crystal exhibits two $\{111\}$ faces and elongates along the $<100>$ direction.

screened for regions that are expected to be located in the magnetosome lumen (i.e., the membrane compartment where MNP synthesis is taking place and that contain a high number of amino acids carrying charged and hydroxyl side chains). These amino acids are expected to be involved in magnetite-binding and iron biomineralization. ${ }^{12,30,31}$ A sequence region close to the C-terminus of Mad10 fits these criteria.

A comparative genomic analysis with all sequenced MTB that produce bullet-shaped MNPs (Table S1) shows that all species have a copy of mad10. This result reinforces the putative involvement of Mad10 in the formation of bullet-shaped MNPs. An amino acid sequence alignment (Figure 2) as well as a Mad10-based phylogenetic analysis (Figure S1) indicate that this protein is well-conserved among bullet-shaped producers of the Proteobacteria and Nitrospirae phyla. Structure prediction algorithms suggest that Mad10 from Desulfamplus magnetovallimortis BW-1 possess three $\alpha$-helical regions (two of which are conserved among all investigated Mad10 proteins). A highly conserved region of Mad10 is located close to the C-terminus and corresponds to one of the predicted helices (amino acids 74-99). This helix contains a specific pattern of hydrophobic and charged amino acids, which aligns them on specific faces of the helix. This further supports the hypothesis that this region is involved in the interaction with magnetite. ${ }^{12}$ Mad10 was therefore identified as the most promising magnetite-binding protein. The biological function of this small protein (113 amino acids) is currently unknown. It contains a CXXC magnetochrome-like motif $^{38}$ at the $\mathrm{C}$-terminus, suggesting that it may also be involved in redox control.

Considering the limited structural and functional information available for Mad10, we took a reductionist approach and focused our characterization on a synthetic peptide fragment (Mad10p; amino acids 72-102) that is slightly longer than the conserved C-terminal helix (Figure 3a). The predicted helical part was extended by two charged amino acids at the $\mathrm{N}$ terminus, which may potentially be involved in the magnetite interaction. Three amino acids were further added at the Cterminus to ensure that all possible structurally and functionally relevant amino acids are included, which are present $\mathrm{N}$ terminally of a helix-breaking proline at position 103 (Figure 2 ). We envisioned that focusing on the putative magnetitebinding sequence facilitates a straightforward binding analysis, avoiding recombinant expression of this unknown protein. A synthetic peptide fragment of the protein Mms6 was already used for the synthesis of magnetite nanoparticles. Synthetic magnetite binding peptides are thus also of interest for applications. ${ }^{16}$ From a practical point of view, the use of the peptide fragment Mad10p further enabled us to prepare a 


$\begin{array}{lllll} & & & 10 & 20\end{array}$

$\begin{array}{ll}\mathbf{1} & (\mathrm{CCO} 06692) \\ \mathbf{2} & (\mathrm{KPA14282}) \\ \mathbf{3} & (\mathrm{AFZ77033)} \\ \mathbf{4} & \\ \mathbf{5} & (\text { WP_015862713) } \\ \mathbf{6} & (\text { WP_040335342) } \\ \mathbf{7} & (\text { KJU } 87659) \\ \mathbf{8} & (\text { AMP41553) } \\ \mathbf{9} & (\text { AMP41633) } \\ \mathbf{1 0} & (\text { AMP41535) } \\ \mathbf{1 1} & (\text { KWT94828) } \\ \mathbf{1 2} & (\text { KJR42670) }\end{array}$

\begin{tabular}{l|cccc}
80 & 90 & 100 & 110 \\
FKDDELSKLFSEIDAINRELDSYIEERDEILYPTGRSVCSQCA---- \\
FQDRDLTNLFRRIDELESRIERRLQERESRLYPNGFSQPVPAAA--- \\
FSDDDMVDLFREFDRVDEDLARARKEREERLYPGLRTTEQEA----- \\
FSDDDMVDLFREFDRVDEDLARARREREERLYPGLRTTEQEA----- \\
FSDEDLSQLFVEFDKADAELSQAKQEREARLYPRSEASAQPA----- \\
ARDDELTRLLDEFDDIQNKAETFLKERTERLYPQEATSCVHSAA//- \\
ARDETLSSLLEEFDAVEGKADTLLKERTERLYP----------- \\
SRDEELTRLFEEFDDVAGRTEALLKDRNQRLYPS-TSACSGEEA//- \\
ARDETLSSLLEEFDAVEGKAETLLKERTERLYPTGAASCVPTTT//- \\
AQDEKLKALLEKFDALEDKKSLFVAERDKLLYPEKKAAEPVMAH / /- \\
FEDENIREILAALDETEKKYDEFVAERQSRLSSASTCSCSGTLD//- \\
SQNEEVISMLNDINELDNKRTNLINERKQRLHKKERTWFAKCKDKC-
\end{tabular}

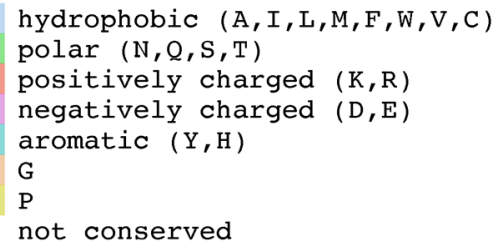

a-helix (Jpred4 for CCO06692)

Figure 2. Alignment of conserved regions of the amino acid sequences of Mad10 proteins found in MTB. Twelve sequences were retrieved from databases: 1. Desulfamplus magnetovallimortis BW- $1,{ }^{22} 2$. Candidatus Magnetomorum sp. HK-1, ${ }^{32}$ 3. Desulfonatronum sp. ML- $1,{ }^{33} 4$. Desulfonatronum sp. ZZ-1, ${ }^{33}$ 5. Desulfovibrio magneticus RS- $1,{ }^{34}$ 6. Candidatus Magnetobacterium casensis, ${ }^{35}$ 7. Candidatus Magnetobacterium bavaricum, ${ }^{36} 8$. uncultured Nitrospirae bacterium 1, 9. uncultured Nitrospirae bacterium 2, 10. uncultured Nitrospirae bacterium 3, 11. Nitrospirae bacterium HCH-1 and 12. Candidatus Magnetoovum chiemensis. ${ }^{37}$ The numbering of amino acids corresponds to the sequence of Desulfamplus magnetovallimortis BW-1. The black boxes represent regions predicted to be helical, using Jpred4 on the sequence of Desulfamplus magnetovallimortis BW-1.

scrambled peptide sequence (Mad10sc; Figure 3a) for probing the specificity of the interaction with epitaxially grown magnetite thin films, exhibiting $\mathrm{Fe}_{3} \mathrm{O}_{4}(100)$ or $\mathrm{Fe}_{3} \mathrm{O}_{4}(111)$ crystal faces (Figure $3 \mathrm{~b}$; see Supporting Information for the synthesis and further characterization of the thin films; Figures S2-S4). When comparing the circular dichroism (CD) spectra of both peptides in the presence and absence of $\mathrm{Fe}^{2+}$ ions, Mad10p folded into an $\alpha$-helical structure in the presence of $\mathrm{Fe}^{2+}$ ions (Figure S5). This does not only support the results of the secondary structure prediction. It also confirms our hypothesis that this region of the protein is able to interact with magnetite.

Specificity of the Mad10p-Magnetite Interaction. In a first series of SMFS experiments, we tested the specificity of the interaction of Mad10p and Mad10sc with $\mathrm{Fe}_{3} \mathrm{O}_{4}(100)^{39-41}$ and $\mathrm{Fe}_{3} \mathrm{O}_{4}(111)$ thin films. The peptides were covalently coupled to the AFM cantilever in a site-specific manner using a poly(ethylene glycol) (PEG) linker (Figure 3a), ${ }^{42}$ and allowed to interact with the different surfaces. Cantilevers functionalized only with the PEG linker were used as an additional negative control. Typical force-extension curves for the Mad10pmagnetite interaction are shown in Figure 3. For all configurations, the data was analyzed to count the number of force-extension curves that showed no, one, or multiple (i.e., two or more) rupture events (Figure S6 and Table S2). The results are summarized in Table 1 . The results show a higher interaction frequency (one or multiple rupture events) for Mad10p when compared with Mad10sc and the PEG linker only. For the peptide-free control, binding was almost completely absent ( $\leq 5 \%$ on both surfaces), whereas a slightly higher interaction frequency of $6-10 \%$ was observed for Mad10sc. This is not unexpected as this peptide also contains a large number of charged amino acids that can potentially interact with magnetite. ${ }^{12,30,31}$ Together, this result clearly suggests that Mad10p binds to both magnetite surfaces in a specific manner. The specificity of binding was further confirmed in additional control experiments where the interaction of Mad10p with glass and mica surfaces was tested (Table S3).

Kinetic Analysis of the Mad10p-Magnetite Interaction. For a more detailed binding analysis, we focused only on force-extension curves that displayed a single rupture event. The rupture forces were extracted, and rupture force histograms were generated. For both $\mathrm{Fe}_{3} \mathrm{O}_{4}(100)$ and $\mathrm{Fe}_{3} \mathrm{O}_{4}(111)$ surfaces, the rupture force histograms (Figure 4 and Figure S7) are broad and cannot be fitted with a Gaussian distribution, which is frequently used to obtain the most probable rupture force. Similarly broad rupture force distributions have been observed in earlier studies, when investigating other interactions between biomolecules and inorganic surfaces. ${ }^{26,28}$ These broad distributions are frequently assigned to the presence of multiple rupture events. $^{26,43}$ If two or more biomolecules dissociate from the surface simultaneously, the additional rupture events may not be visible in the force-extension curve, while a higher rupture force may still be detected. ${ }^{44}$ In our case, we exclude this possibility as the long PEG spacer used (controur length $>60 \mathrm{~nm}^{45}$ ) decreases the probability that two peptides detach from the surface at exactly the same extension.

Assuming that a small number of peptides immobilized at the tip of the cantilever possess an equal chance to independently interact with the surface, we further validated the selection of single rupture events with a theoretical Poisson distribution. Assuming a given number of no rupture events (which are most accurately identified), we calculated the expected number of single and multiple rupture events. Given that the selected 
a

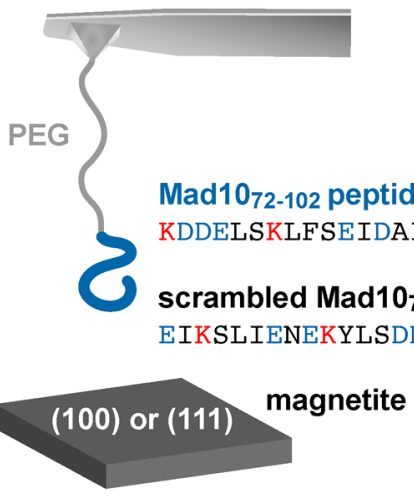

b
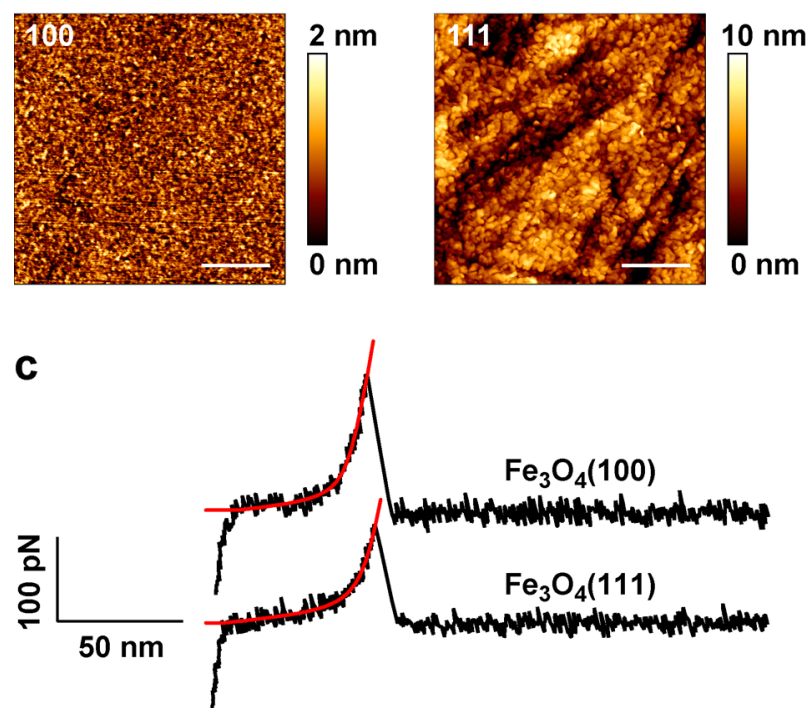

Figure 3. AFM-based single-molecule force spectroscopy (SMFS) for investigating the interaction between a peptide fragment of the putative magnetite-binding protein Mad10 (Mad10p) and magnetite $\mathrm{Fe}_{3} \mathrm{O}_{4}(100)$ and $\mathrm{Fe}_{3} \mathrm{O}_{4}(111)$ thin films. (a) SMFS setup, including the amino acid sequence of Mad10p and the corresponding scrambled sequence Mad10sc. The peptides are immobilized to the AFM cantilever via long (10 kDa; > $60 \mathrm{~nm})$ PEG spacers, using thiol-maleimide chemistry. The peptides carry a cysteine residue at their C-terminus. (b) AFM images of epitaxially grown $\mathrm{Fe}_{3} \mathrm{O}_{4}(100)$ and $\mathrm{Fe}_{3} \mathrm{O}_{4}(111)$ thin films (scale bar: $500 \mathrm{~nm}$ ). (c) Typical force-extension curves showing a single rupture event. The red lines are fits to the extensible freely jointed chain (eFJC) model (see Supporting Information).

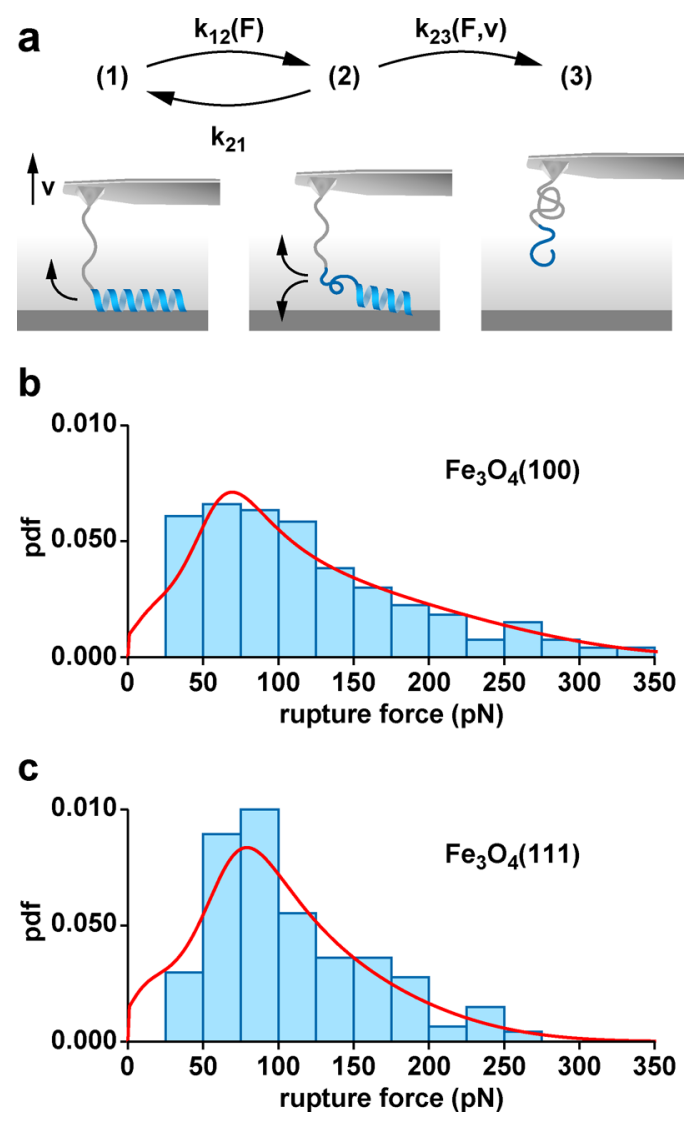

Figure 4. Three-state model to describe the Mad10p-magnetite interaction. (a) Scheme of the three-state model. From the strongly bound state (1) the peptide unbinds to a weakly associated state (2) with the force-dependent unbinding rate $k_{12}(\mathrm{~F})$. From this state, the peptide either rebinds to state (1) with rate $k_{21}$ or completely dissociates from the surface with a force- and speed-dependent rate $k_{23}(\mathrm{~F}, \mathrm{v})$. (b) Probability density function (pdf) of the rupture forces for the Mad10p $-\mathrm{Fe}_{3} \mathrm{O}_{4}(100)$ interaction, measured at a retraction speed of $1 \mu \mathrm{m} \mathrm{s}^{-1}(n=479)$. Adjusting the numerical values of the free model parameters, the theoretical probability density function (red line) describes the experimental data for specific parameter values (Table 2). (c) Probability density function of the rupture forces for the Mad10p- $-\mathrm{Fe}_{3} \mathrm{O}_{4}(111)$ interaction, measured at a retraction speed of $1 \mu \mathrm{m} \mathrm{s}^{-1}(n=188)$. The red line represents the theoretical probability density function obtained from our model. The respective parameter values are given in Table 2.

number of single rupture events lies below the fraction predicted from the Poisson distribution, we infer that it is highly unlikely that multiple ruptures are hidden inside the population of single rupture events. We thus conclude that the broad rupture force distributions describing the Mad10p-magnetite interaction do

Table 1. Specificity of the Mad10p-Magnetite Interaction ${ }^{a}$

\begin{tabular}{|c|c|c|c|c|c|c|}
\hline & \multicolumn{6}{|c|}{ relative number of rupture events in \% } \\
\hline & \multicolumn{3}{|c|}{$\mathrm{Fe}_{3} \mathrm{O}_{4}(100)$} & \multicolumn{3}{|c|}{$\mathrm{Fe}_{3} \mathrm{O}_{4}(111)$} \\
\hline & 0 & 1 & $\geq 2$ & 0 & 1 & $\geq 2$ \\
\hline Mad10p & $81(81)$ & $9(17)$ & $10(2)$ & $58(58)$ & $9(32)$ & $33(10)$ \\
\hline Mad10sc & $90(90)$ & $1(9)$ & $9(1)$ & $94(94)$ & $1(6)$ & $5(0)$ \\
\hline PEG only & $95(95)$ & $5(5)$ & $0(0)$ & $98(98)$ & $2(2)$ & $0(0)$ \\
\hline
\end{tabular}

${ }^{a_{T}}$ The data was collected at a cantilever retraction speed of $1 \mu \mathrm{m} \mathrm{s}^{-1}$. The data is reported as the relative amount of zero (0), single (1) and multiple $(\geq 2)$ rupture events. The total number of force-extension curves and the amount of cantilevers used for each condition is reported in Table S2 in the supporting information. The values in brackets represent the expected number of rupture events calculated using a Poisson distribution. 
not originate from multiple rupture events but are instead a characteristic of this specific Mad10p-magnetite interaction.

We hypothesize that these broad force distributions originate from one of the following three possibilities: the first possibility is the inherent heterogeneity originating from the magnetite thin films in the form of defects or different crystal faces. AFM height images of the magnetite surfaces confirm the presence of defects and irregular nanoscale features (Figure 3b, Figure S3). Mad10p potentially binds with a different conformation and thus affinity to the local crystalline surface it interacts with. The second possibility is a conformational heterogeneity of the peptide itself. We exclude this possibility, as the $\alpha$-helical conformation of the peptide is stabilized by iron binding, as proven by $\mathrm{CD}$ spectroscopy (Figure S5). The third possibility is rebinding of the peptide to the surface. A crystalline surface exhibits a very high density of possible binding sites so that the probability of rebinding (i.e., the association rate) can be much higher than for receptor-ligand interactions, which are usually investigated with SMFS.

To infer the possible contribution of rebinding, we further characterized the nature of the Mad10p-magnetite interaction in a dynamic SMFS experiment where the interaction is probed at different retraction speeds (i.e., loading rates $r=\mathrm{d} F / \mathrm{d} t$ ). In such an experiment, rebinding is more likely at low retraction speeds and becomes less and less probable with increasing retraction speeds. The most frequently used Bell-Evans model, which does not include the possibility of rebinding, predicts that the rupture force distribution shifts to higher values with increasing retraction speed. ${ }^{46-48}$ In contrast to this prediction, all rupture force histograms look almost identical and are hardly shifted from one retraction speed to another, suggesting that the rupture forces are independent of the retraction speed. Furthermore, the mean rupture force seems to be independent of the retraction speed in the range of $0.05 \mu \mathrm{m} \mathrm{s}^{-1}$ to $10 \mu \mathrm{m} \mathrm{s}^{-1}$. Only at the highest retraction speed of $16 \mu \mathrm{m} \mathrm{s}^{-1}$, corresponding to a mean loading rate of about $10^{5} \mathrm{pN} \mathrm{s}^{-1}$, we observe a slight increase in the mean rupture force (Figure S8). This finding suggests that the system is in a quasi-equilibrium at retraction speeds up to $10 \mu \mathrm{m} \mathrm{s}^{-1}$, indicating that the peptide rebinds to the surface while the cantilever is retracted. ${ }^{28,48-50}$

For further analysis, we calculated the effective forcedependent unbinding rates from the rupture force histograms using the method of Dudko et al. ${ }^{51}$ (see Supporting Information). The unbinding rates exhibit an unexpected dependence on the retraction speed (Figure S9a,b). They become faster with increasing retraction speed for any given force. At the same time, the functional dependence on the force seems to be insensitive to the speed, as if the retraction speed would determine an overall rescaling of the force-dependent unbinding rate. Such a behavior is in sharp contrast with a simple bond model, where the force-dependent unbinding rate is independent of the retraction speed. As predicted by Dudko and co-workers, ${ }^{52}$ the unbinding rates obtained from measurements at different retraction speeds collapse onto one force-dependent curve, which is not the case for the data shown here. As a consequence, neither the Bell-Evans nor the Dudko method can be directly applied to extract kinetic information about the peptide-surface interactions. With the aim of fully describing the obtained force distributions and of obtaining kinetic information, we therefore introduce a theoretical description that includes the possibility of rebinding (Figure 4a; see SI for a full description of the model and the corresponding data analysis).
Our theory to describe unbinding from the surface is based on a three-state model: in state (1), the peptide is strongly bound to the surface, in state (2) it is closely associated with the surface, but not strongly bound, and in state (3), the peptide is completely dissociated (Figure 4a). We assume that the transition rate from the bound state (1) to the closely associated intermediate state (2) depends exponentially on the applied force as $k_{12}(F)=k_{12}^{0} \exp \left(F x_{1} / k_{B} T\right)$, where $k_{12}^{0}$ is the force-free unbinding rate, $x_{1}$ the distance from the bound state to the transition state, and $k_{\mathrm{B}} T$ the thermal energy. From the intermediate state (2), the peptide can either rebind to the surface (1) with the constant, force-independent rate $k_{21}$ or dissociate completely (3) with rate $k_{23}$. However, in the intermediate state, the peptide is pulled away from the surface with constant speed, so that rebinding is only possible within a time window that becomes shorter with increasing retraction speed. We therefore assume that the transition rate from the intermediate to the unbound state is linearly proportional to the retraction speed, while it also depends on the force exponentially as $k_{23}(F, v)=\chi^{-1}\left(v_{0}+v\right) \exp \left(F x_{2} / k_{\mathrm{B}} T\right)$, where $\chi$ is an interaction distance, $v_{0}$ a velocity offset, $x_{2}$ the distance from the bound state to the transition state, and $k_{\mathrm{B}} T$ the thermal energy. To obtain the probability density function of the rupture forces, we transform a master equation that describes the time evolution of the probability $p_{i}$ for the system to be in one of the three states (i) into a function of force:

$$
\begin{aligned}
& \frac{\mathrm{d} p_{1}(F)}{\mathrm{d} F}=\left(\frac{\mathrm{d} F}{\mathrm{~d} t}\right)^{-1}\left(-k_{12}(F) p_{1}(F)+k_{21} p_{2}(F)\right) \\
& \frac{\mathrm{d} p_{2}(F)}{\mathrm{d} F}=\left(\frac{\mathrm{d} F}{\mathrm{~d} t}\right)^{-1}\left(k_{12}(F) p_{1}(F)-\left(k_{21}+k_{23}(F, v)\right) p_{2}(F)\right) \\
& \frac{\mathrm{d} p_{3}(F)}{\mathrm{d} F}=\left(\frac{\mathrm{d} F}{\mathrm{~d} t}\right)^{-1} k_{23}(F, v) p_{2}(F)
\end{aligned}
$$

These equations involve the loading rate $\mathrm{d} F / \mathrm{d} t$ that depends on the retraction speed and the nonlinear elastic properties of the PEG linker (see Supporting Information for details). The last equation (eq 3) provides the probability density function of the rupture forces.

The numerical values of the model parameters were chosen such that the model matches the experimental rupture force histograms for a retraction speed of $1 \mu \mathrm{m} \mathrm{s}^{-1}$ (Figure $4 \mathrm{~b}, \mathrm{c}$, Table 2 , and Figure S10). With these parameter values, the model is also in quantitative agreement with the measured rupture force histograms for all other retraction speeds (Figure S11). The calculated distributions are almost independent of the retraction speed. Moreover, the model allows us to obtain an explicit analytical expression for the effective force-dependent unbinding rate. The calculated unbinding rate agrees very well with the experimental data (Figure S9). Specifically, it reproduces the unusual dependence on the retraction speed.

The kinetic parameters obtained from this three-state model indicate a very large rebinding rate $k_{21}$ of the peptide to both surfaces (9900 s $\mathrm{s}^{-1}$ for the $\mathrm{Fe}_{3} \mathrm{O}_{4}(100)$ and $9000 \mathrm{~s}^{-1}$ for the $\mathrm{Fe}_{3} \mathrm{O}_{4}(111)$ surfaces, respectively; Table 2). Thus, the probability that the peptide rebinds to the surface upon initial unbinding is very close to 1 for small forces and small retraction speeds (Figures S9c,d). In addition, the model can be used to estimate an effective load-free unbinding rate $k_{\text {off }}=\chi^{-1} v_{0} k_{12}^{0} /$ $\left(\chi^{-1} v_{0}+k_{12}^{0}+k_{21}\right)$, which is $4 \times 10^{-4} \mathrm{~s}^{-1}$ for the $\mathrm{Fe}_{3} \mathrm{O}_{4}(100)$ and 
Table 2. Numerical Values of the Model Parameters Describing the Rupture Force Histograms for the $\mathrm{Fe}_{3} \mathrm{O}_{4}(100)$ and $\mathrm{Fe}_{3} \mathrm{O}_{4}(111)$ Surfaces ${ }^{a}$

\begin{tabular}{lccccc} 
& \multicolumn{5}{c}{ model parameters } \\
\cline { 2 - 6 } & $k_{12}^{0}\left(\mathrm{~s}^{-1}\right)$ & $k_{21}\left(\mathrm{~s}^{-1}\right)$ & $x_{1}(\mathrm{~nm})$ & $\chi(\mathrm{nm})$ & $x_{2}(\mathrm{~nm})$ \\
$\mathrm{Fe}_{3} \mathrm{O}_{4}(100)$ & 80 & 9900 & 0.350 & 18.181 & 0.026 \\
$\mathrm{Fe}_{3} \mathrm{O}_{4}(111)$ & 77 & 9000 & 0.299 & 13.158 & 0.028
\end{tabular}

${ }^{a}$ The parameters were determined for one data set each (measured at a retraction speed of $1 \mu \mathrm{m} \mathrm{s}^{-1}$ ) and validated with additional data sets (see Supporting Information). The numerical parameters for the nonlinear elasticity of the PEG linker are given in the Supporting Information. For the thermal energy, we use the value $k_{\mathrm{B}} T=$ $4.1 \mathrm{pN} \mathrm{nm}$ and for the velocity offset $v_{0}=1 \mathrm{~nm} \mathrm{~s}^{-1}$.

$6 \times 10^{-4} \mathrm{~s}^{-1}$ for the $\mathrm{Fe}_{3} \mathrm{O}_{4}(111)$ surface, respectively. In combination, this demonstrates a very strong interaction between Mad10p and the magnetite surface-again, however, with only a small difference between the different crystal faces when all model parameters are compared (Table 2).

As briefly mentioned above, broad rupture force distributions can also result from heterogeneities, either on the surface or in the peptide conformation. To test this possibility, we have further tested an alternative model that considers different types of bonds that can form between the peptide and the surfaces. This model assumes that each type of bond possesses a different stability and that its mechanical dissociation follows the BellEvans model ${ }^{46}$ (see Supporting Information for details). Although such a description can account for the rupture force distribution of one data set measured at one given retraction speed, it fails to reproduce the insensitivity of the complete set of rupture force distributions measured over a range of retraction speeds (Figure S12). We therefore conclude that the rebinding model is more consistent with the experimental data. Overall, considering the small effective load-free unbinding rate on the order of $10^{-4} \mathrm{~s}^{-1}$, we conclude that Mad10p is bound to the magnetite surface very strongly. According to the kinetic model, strong binding in the absence of force results from rapid exchange between the bound and intermediate states, with the equilibrium strongly shifted toward the bound state.

\section{DISCUSSION}

The data presented above show that the conserved C-terminal $\alpha$-helix of the protein Mad10 (Mad10p) binds to magnetite specifically and with high affinity; however, no crystal-facespecificity was observed. Because of the apparent lack of crystalface-specificity, we exclude the possibility that Mad10 plays an essential role in breaking the symmetry during magnetite crystal growth. It should be noted, however, that the thin films are not atomically flat and may expose other crystal faces (Figure S3), which may prohibit the detection of crystal-face-specific interactions. Independent of this inherent experimental limitation, the strong binding of Mad10p to magnetite allows us to conclude that the protein Mad10 is involved in the formation of magnetite crystals. The strong binding to magnetite surfaces results from rapid rebinding upon initial dissociation, which makes binding almost irreversible in the absence of force. This interaction with magnetite surfaces is most likely driven by acidic amino acids. Five aspartic acid and six glutamic acid residues are arranged in a repetitive pattern within the sequence of Mad10p, and these amino acids are known to interact with magnetite. Molecular dynamics simulations have, for example, shown that these amino acids bind to the magnetite (111) crystal face. ${ }^{30}$ In addition, infrared spectroscopy and X-ray photoelectron spectroscopy experiments have proven the interaction of glutamic acid with magnetite. ${ }^{31} \mathrm{~A}$ defined arrangement of these two amino acids was also observed for the magnetosomal proteins Mms6 ${ }^{12,14,16}$ and MamC. ${ }^{53}$ Hence, the conserved Mad10p region could be the key binding site to magnetite. In combination with the observation that trace amounts of iron ions are required to stabilize the $\alpha$-helix in solution, this leads to the hypothesis that ion binding stabilizes the secondary structure by binding to regularly spaced aspartic acid and glutamic acid residues.

The reduced number of rupture events measured for Mad10sc highlights that both the amino acid composition and the regular arrangement of these amino acids on the surface of an $\alpha$-helix are important factors for determining the peptide-magnetite interaction. Besides the regular arrangement of acidic residues, the helix further displays hydrophobic residues concentrated on one face of the $\alpha$-helix. The resulting amphiphilic nature of the Mad10p $\alpha$-helix may facilitate the interaction of the hydrophobic region with the native environment of the magnetosome membrane, ${ }^{54-56}$ with other structural elements of Mad10 or also other magnetosome-associated proteins. Nucleation of bacterial magnetite crystals is hypothesized to occur at the membrane surface, and proteins are considered to act as nucleation templates. ${ }^{57}$ Nucleation at surfaces was also observed in other biomineralization processes. ${ }^{58}$ Considering its ability to bind cations and its putative interaction with the magnetosome membrane, we thus propose that Mad10 is involved in the nucleation of magnetite crystals. To confirm this hypothesis, the full-length Mad10 protein needs to be investigated in vitro to characterize its overall structure and its putative role in magnetite biomineralization. Also, in vivo knockout or overexpression studies of this protein will shed more light on its biological function. The recent development of a genome editing method for Desulfovibrio magneticus strain RS-1, ${ }^{59}$ a magnetotatic bacterium producing similar MNPs as BW-1, will open up new strategies for the characterization of Mad proteins.

The strong binding of Mad10p to magnetite presents a highly powerful starting point for tailoring the newly discovered magnetite-binding protein Mad10 for biomedical and biotechnological applications, such as previously reported using the magnetosome protein MamC. ${ }^{60}$ Fusing Mad10p (or the fulllength protein) to any protein of interest, will allow for specifically labeling MNPs in a one-pot reaction without the need of any additional linker. Mad10p can thus act as a magnetite-binding domain for attaching different fluorophores or biomolecules (e.g., recombinant antibodies) to magnetite surfaces. This strategy may also be implemented for the immobilization of affinity ligands for protein purification, making use of the magnetic behavior of MNPs. ${ }^{61}$ Furthermore, Mad10p may be developed into a coating for MNPs, blocking the nonspecific interaction of other proteins. Such coatings might be of great interest for biomedical MNP applications, such as hyperthermia treatments or targeted drug delivery. An additional application of Mad10p could be the production of particle-reinforced composites. When coupling Mad10p to branched polymers, such as 4-arm poly(ethylene glycol), hydrogel formation can be induced upon adding MNPs. Such materials were, for example, obtained with MNPs and DOPA (L-3,4-dihydroxyphenylalanine)-functionalized polymers. ${ }^{62}$

In conclusion, we identified a new magnetite-binding protein, named Mad10. This protein is derived from the magnetotactic bacterium Desulfamplus magnetovallimortis strain BW-1, which 
mineralizes bullet-shaped magnetite crystals. Using SMFS, we show that the highly conserved C-terminal region of Mad10 specifically and strongly binds to magnetite thin films but does not display crystal-face-specificity. This strong peptide-magnetite interaction is characterized by broad rupture force distributions that do not depend on the retraction speed of the AFM cantilever. Our theory suggests that these broad rupture force distributions, insensitive to the retraction speed, originate from an unbinding process that involves a weakly associated state with a speed-dependent lifetime. From this state, the peptide can either rebind to a strongly associated state or dissociate from the surface with a speed-dependent rate. Utilizing this strong interaction, we envision that this new magnetite-binding peptide Mad10p can be used for the functionalization of magnetite nanoparticles in biotechnological and biomedical applications.

\section{ASSOCIATED CONTENT}

\section{S Supporting Information}

The Supporting Information is available free of charge on the ACS Publications website at DOI: 10.1021/acs.nanolett.9b03560.

Experimental protocols, detailed results of the dynamic force spectroscopy experiments, and description of the theoretical models (PDF)

\section{AUTHOR INFORMATION}

\section{Corresponding Authors}

*E-mail: kerstin.blank@mpikg.mpg.de.

*E-mail: damien.faivre@mpikg.mpg.de.

\section{ORCID $\odot$}

Ruby M. A. Sullan: 0000-0002-8927-2605

Nika Spiridis: 0000-0002-5276-0568

Stefan Klumpp: 0000-0003-0584-2146

Kerstin G. Blank: 0000-0001-5410-6984

Damien Faivre: 0000-0001-6191-3389

\section{Present Addresses}

-A.P.: Department of Chemical and Environmental Engineering, University of California at Riverside, 900 University Ave., Riverside, CA 92521, United States

R.M.A.S.: Department of Physical and Environmental Sciences, University of Toronto Scarborough, 1265 Military Trail, Toronto, M1C 1A4, Canada

C.V.-T.: Instituto de Investigaciones Biomédicas y Sanitarias (IUIBS), Universidad de Las Palmas de Gran Canaria, 35016 Las Palmas de Gran Canaria, Spain

\section{Author Contributions}

Conceptualization, S.K., K.G.B., and D.F.; Investigation, A.P., R.M.A.S, C.V.T. K.F., N.S., C.T.L., and N.M.; Formal Analysis, A.P., R.M.A.S., F.B,. and S.K.; Resources, K.F., N.S., C.T.L. N.M., K.G.B., and D.F.; Writing - Original Draft, A.P., R.M.A.S., F.B., and C.V.T.; Writing - Review \& Editing, C.T.L., S.K., K.G.B., and D.F.; Funding Acquisition, R.M.A.S., F.B., K.G.B., and D.F.; Supervision, S.K., K.G.B., and D.F. All authors have given approval to the final version of the manuscript.

\section{Author Contributions}

*(A.P., F.B., R.M.A.S.) These authors contributed equally.

\section{Notes}

The authors declare no competing financial interest.
Raw data of the SMFS experiments have been deposited on Edmond - the Open Access Data Repository of the Max Planck Society (https://edmond.mpdl.mpg.de).

\section{ACKNOWLEDGMENTS}

This work was supported by the Max Planck Society and the International Max Planck Research School (IMPRS) on Multiscale Bio-Systems. R.M.A.S. and F.B. thank the Alexander von Humboldt foundation for their Postdoctoral Research Fellowships.

\section{REFERENCES}

(1) Prozorov, T.; Bazylinski, D. A.; Mallapragada, S. K.; Prozorov, R. Novel magnetic nanomaterials inspired by magnetotactic bacteria: Topical review. Mater. Sci. Eng., R 2013, 74, 133-172.

(2) Faivre, D.; Godec, T. U. From Bacteria to Mollusks: The Principles Underlying the Biomineralization of Iron Oxide Materials. Angew. Chem., Int. Ed. 2015, 54, 4728-4747.

(3) Wang, J.; Cheng, Q.; Tang, Z. Layered nanocomposites inspired by the structure and mechanical properties of nacre. Chem. Soc. Rev. 2012, 41, 1111-1129.

(4) Wegst, U. G. K.; Bai, H.; Saiz, E.; Tomsia, A. P.; Ritchie, R. O. Bioinspired structural materials. Nat. Mater. 2015, 14, 23-36.

(5) Cantaert, B.; Kuo, D.; Matsumura, S.; Nishimura, T.; Sakamoto, T.; Kato, T. Use of Amorphous Calcium Carbonate for the Design of New Materials. ChemPlusChem 2017, 82, 107-120.

(6) Thevenot, P.; Hu, W.; Tang, L. Surface chemistry influences implant biocompatibility. Curr. Top. Med. Chem. 2008, 8, 270-280.

(7) Fenoglio, I.; Fubini, B.; Ghibaudi, E. M.; Turci, F. Multiple aspects of the interaction of biomacromolecules with inorganic surfaces. Adv. Drug Delivery Rev. 2011, 63, 1186-1209.

(8) Pagel, M.; Beck-Sickinger, A. G. Multifunctional biomaterial coatings: synthetic challenges and biological activity. Biol. Chem. 2017, $398,3-22$.

(9) Lefevre, C. T.; Trubitsyn, D.; Abreu, F.; Kolinko, S.; de Almeida, L. G. P.; de Vasconcelos, A. T. R.; Lins, U.; Schüler, D.; Ginet, N.; Pignol, D.; Bazylinski, D. A. Monophyletic origin of magnetotaxis and the first magnetosomes. Environ. Microbiol. 2013, 15, 2267-2274.

(10) Pósfai, M.; Lefèvre, C.; Trubitsyn, D.; Bazylinski, D.; Frankel, R. Phylogenetic significance of composition and crystal morphology of magnetosome minerals. Front. Microbiol. 2013, 4, 344.

(11) Uebe, R.; Schüler, D. Magnetosome biogenesis in magnetotactic bacteria. Nat. Rev. Microbiol. 2016, 14, 621-637.

(12) Arakaki, A.; Webb, J.; Matsunaga, T. A. Novel Protein Tightly Bound to Bacterial Magnetic Particles in Magnetospirillum magneticum Strain AMB-1. J. Biol. Chem. 2003, 278, 8745-8750.

(13) Li, J.; Menguy, N.; Gatel, C.; Boureau, V.; Snoeck, E.; Patriarche, G.; Leroy, E.; Pan, Y. Crystal growth of bullet-shaped magnetite in magnetotactic bacteria of the Nitrospirae phylum. J. R. Soc., Interface 2015, 12, 20141288.

(14) Amemiya, Y.; Arakaki, A.; Staniland, S. S.; Tanaka, T.; Matsunaga, T. Controlled formation of magnetite crystal by partial oxidation of ferrous hydroxide in the presence of recombinant magnetotactic bacterial protein Mms6. Biomaterials 2007, 28, 53815389.

(15) Prozorov, T.; Mallapragada, S. K.; Narasimhan, B.; Wang, L.; Palo, P.; Nilsen-Hamilton, M.; Williams, T. J.; Bazylinski, D. A.; Prozorov, R.; Canfield, P. C. Protein-Mediated Synthesis of Uniform Superparamagnetic Magnetite Nanocrystals. Adv. Funct. Mater. 2007, 17, 951-957.

(16) Arakaki, A.; Masuda, F.; Amemiya, Y.; Tanaka, T.; Matsunaga, T. Control of the morphology and size of magnetite particles with peptides mimicking the Mms6 protein from magnetotactic bacteria. J. Colloid Interface Sci. 2010, 343, 65-70.

(17) Tanaka, M.; Mazuyama, E.; Arakaki, A.; Matsunaga, T. MMS6 Protein Regulates Crystal Morphology during Nano-sized Magnetite Biomineralization in Vivo. J. Biol. Chem. 2011, 286, 6386-6392. 
(18) Muxworthy, A. R.; Williams, W. Critical single-domain/ multidomain grain sizes in noninteracting and interacting elongated magnetite particles: Implications for magnetosomes. J. Geophys. Res.: Solid Earth 2006, 111, B12S12.

(19) Körnig, A.; Winklhofer, M.; Baumgartner, J.; Gonzalez, T. P.; Fratzl, P.; Faivre, D. Magnetite Crystal Orientation in Magnetosome Chains. Adv. Funct. Mater. 2014, 24, 3926-3932.

(20) Mériaux, S.; Boucher, M.; Marty, B.; Lalatonne, Y.; Prévéral, S.; Motte, L.; Lefêvre, C. T.; Geffroy, F.; Lethimonnier, F.; Péan, M.; Garcia, D.; Adryanczyk-Perrier, G.; Pignol, D.; Ginet, N. Magnetosomes, Biogenic Magnetic Nanomaterials for Brain Molecular Imaging with 17.2 T MRI Scanner. Adv. Healthcare Mater. 2015, 4, 1076-1083. (21) Lefêvre, C. T.; Menguy, N.; Abreu, F.; Lins, U.; Pósfai, M.; Prozorov, T.; Pignol, D.; Frankel, R. B.; Bazylinski, D. A. A Cultured Greigite-Producing Magnetotactic Bacterium in a Novel Group of Sulfate-Reducing Bacteria. Science 2011, 334, 1720-1723.

(22) Lefêvre, C. T.; Trubitsyn, D.; Abreu, F.; Kolinko, S.; Jogler, C.; de Almeida, L. G. P.; de Vasconcelos, A. T. R.; Kube, M.; Reinhardt, R.; Lins, U.; Pignol, D.; Schüler, D.; Bazylinski, D. A.; Ginet, N. Comparative genomic analysis of magnetotactic bacteria from the Deltaproteobacteria provides new insights into magnetite and greigite magnetosome genes required for magnetotaxis. Environ. Microbiol. 2013, 15, 2712-2735.

(23) Lee, H.; Scherer, N. F.; Messersmith, P. B. Single-molecule mechanics of mussel adhesion. Proc. Natl. Acad. Sci. U. S. A. 2006, 103, 12999-13003.

(24) Hayashi, T.; Sano, K.-I.; Shiba, K.; Kumashiro, Y.; Iwahori, K.; Yamashita, I.; Hara, M. Mechanism Underlying Specificity of Proteins Targeting Inorganic Materials. Nano Lett. 2006, 6, 515-519.

(25) Sonnenberg, L.; Luo, Y.; Schlaad, H.; Seitz, M.; Cölfen, H.; Gaub, H. E. Quantitative Single Molecule Measurements on the Interaction Forces of Poly(l-glutamic acid) with Calcite Crystals. J. Am. Chem. Soc. 2007, 129, 15364-15371.

(26) Landoulsi, J.; Dupres, V. Probing Peptide-Inorganic Surface Interaction at the Single Molecule Level Using Force Spectroscopy. ChemPhysChem 2011, 12, 1310-1316.

(27) Razvag, Y.; Gutkin, V.; Reches, M. Probing the Interaction of Individual Amino Acids with Inorganic Surfaces Using Atomic Force Spectroscopy. Langmuir 2013, 29, 10102-10109.

(28) Wei, G.; Li, Q.; Steckbeck, S.; Ciacchi, L. C. Direct force measurements on peeling heteropolymer ssDNA from a graphite surface using single-molecule force spectroscopy. Phys. Chem. Chem. Phys. 2014, 16, 3995-4001.

(29) Maity, S.; Zanuy, D.; Razvag, Y.; Das, P.; Aleman, C.; Reches, M. Elucidating the mechanism of interaction between peptides and inorganic surfaces. Phys. Chem. Chem. Phys. 2015, 17, 15305-15315.

(30) Bürger, A.; Magdans, U.; Gies, H. Adsorption of amino acids on the magnetite-(111)-surface: a force field study. J. Mol. Model. 2013, 19, 851-857.

(31) Schwaminger, S. P.; García, P. F.; Merck, G. K.; Bodensteiner, F. A.; Heissler, S.; Günther, S.; Berensmeier, S. Nature of interactions of amino acids with bare magnetite nanoparticles. J. Phys. Chem. C 2015, 119, 23032-23041.

(32) Kolinko, S.; Richter, M.; Glöckner, F.-O.; Brachmann, A.; Schüler, D. Single-cell genomics reveals potential for magnetite and greigite biomineralization in an uncultivated multicellular magnetotactic prokaryote. Environ. Microbiol. Rep. 2014, 6, 524-531.

(33) Lefèvre, C. T.; Frankel, R. B.; Pósfai, M.; Prozorov, T.; Bazylinski, D. A. Isolation of obligately alkaliphilic magnetotactic bacteria from extremely alkaline environments. Environ. Microbiol. 2011, 13, 23422350.

(34) Nakazawa, H.; Arakaki, A.; Narita-Yamada, S.; Yashiro, I.; Jinno, K.; Aoki, N.; Tsuruyama, A.; Okamura, Y.; Tanikawa, S.; Fujita, N.; Takeyama, H.; Matsunaga, T. Whole genome sequence of Desulfovibrio magneticus strain RS-1 revealed common gene clusters in magnetotactic bacteria. Genome Res. 2009, 19, 1801-1808.

(35) Lin, W.; Deng, A.; Wang, Z.; Li, Y.; Wen, T.; Wu, L.-F.; Wu, M.; Pan, Y. Genomic insights into the uncultured genus 'Candidatus
Magnetobacterium' in the phylum Nitrospirae. ISME J. 2014, 8, 24632477.

(36) Jogler, C.; Wanner, G.; Kolinko, S.; Niebler, M.; Amann, R.; Petersen, N.; Kube, M.; Reinhardt, R.; Schüler, D. Conservation of proteobacterial magnetosome genes and structures in an uncultivated member of the deep-branching Nitrospira phylum. Proc. Natl. Acad. Sci. U. S. A. 2011, 108, 1134-1139.

(37) Kolinko, S.; Richter, M.; Glöckner, F.-O.; Brachmann, A.; Schüler, D. Single-cell genomics of uncultivated deep-branching magnetotactic bacteria reveals a conserved set of magnetosome genes. Environ. Microbiol. 2016, 18, 21-37.

(38) Siponen, M. I.; Legrand, P.; Widdrat, M.; Jones, S. R.; Zhang, W.J.; Chang, M. C. Y.; Faivre, D.; Arnoux, P.; Pignol, D. Structural insight into magnetochrome-mediated magnetite biomineralization. Nature 2013, 502, 681-684.

(39) Korecki, J.; Handke, B.; Spiridis, N.; Ślęzak, T.; Flis-Kabulska, I.; Haber, J. Size effects in epitaxial films of magnetite. Thin Solid Films 2002, 412, 14-23.

(40) Spiridis, N.; Handke, B.; Slezak, T.; Barbasz, J.; Zajac, M.; Haber, J.; Korecki, J. Surface Structure of Epitaxial Magnetite $\mathrm{Fe}_{3} \mathrm{O}_{4}(001)$ Films: In Situ STM and CEMS Studies. J. Phys. Chem. B 2004, 108, 14356-14361.

(41) Spiridis, N.; Barbasz, J.; Łodziana, Z.; Korecki, J. $\mathrm{Fe}_{3} \mathrm{O}_{4}(001)$ films on $\mathrm{Fe}(001)$ : Termination and reconstruction of iron-rich surfaces. Phys. Rev. B: Condens. Matter Mater. Phys. 2006, 74, 155423.

(42) Zimmermann, J. L.; Nicolaus, T.; Neuert, G.; Blank, K. Thiolbased, site-specific and covalent immobilization of biomolecules for single-molecule experiments. Nat. Protoc. 2010, 5, 975-985.

(43) Erdmann, T.; Pierrat, S.; Nassoy, P.; Schwarz, U. S. Dynamic force spectroscopy on multiple bonds: Experiments and model. Europhys. Lett. 2008, 81, 48001.

(44) Getfert, S.; Reimann, P. Hidden Multiple Bond Effects in Dynamic Force Spectroscopy. Biophys. J. 2012, 102, 1184-1193.

(45) Oesterhelt, F.; Rief, M.; Gaub, H. E. Single molecule force spectroscopy by AFM indicates helical structure of poly(ethyleneglycol) in water. New J. Phys. 1999, 1, 6.

(46) Evans, E. Probing the Relation Between Force-Lifetime-and Chemistry in Single Molecular Bonds. Annu. Rev. Biophys. Biomol. Struct. 2001, 30, 105-128.

(47) Evans, E.; Ritchie, K. Dynamic strength of molecular adhesion bonds. Biophys. J. 1997, 72, 1541-1555.

(48) Friddle, R. W.; Noy, A.; De Yoreo, J. J. Interpreting the widespread nonlinear force spectra of intermolecular bonds. Proc. Natl. Acad. Sci. U. S. A. 2012, 109, 13573-13578.

(49) Schönherr, H.; Beulen, M. W. J.; Bügler, J.; Huskens, J.; van Veggel, F. C. J. M.; Reinhoudt, D. N.; Vancso, G. J. Individual Supramolecular Host-Guest Interactions Studied by Dynamic Single Molecule Force Spectroscopy. J. Am. Chem. Soc. 2000, 122, 49634967.

(50) Blass, J.; Albrecht, M.; Wenz, G.; Zang, Y. N.; Bennewitz, R. Single-molecule force spectroscopy of fast reversible bonds. Phys. Chem. Chem. Phys. 2017, 19, 5239-5245.

(51) Dudko, O. K.; Hummer, G.; Szabo, A. Theory, analysis, and interpretation of single-molecule force spectroscopy experiments. Proc. Natl. Acad. Sci. U. S. A. 2008, 105, 15755-15760.

(52) Dudko, O. K.; Hummer, G.; Szabo, A. Intrinsic Rates and Activation Free Energies from Single-Molecule Pulling Experiments. Phys. Rev. Lett. 2006, 96, 108101.

(53) Nudelman, H.; Valverde-Tercedor, C.; Kolusheva, S.; Gonzalez, T. P.; Widdrat, M.; Grimberg, N.; Levi, H.; Nelkenbaum, O.; Davidov, G.; Faivre, D.; et al. Structure-function studies of the magnetitebiomineralizing magnetosome-associated protein MamC. J. Struct. Biol. 2016, 194, 244-252.

(54) Eisenberg, D. Three-dimensional structure of membrane and surface proteins. Annu. Rev. Biochem. 1984, 53, 595-623.

(55) Jackson, M.; Pratt, J. An 18 amino acid amphiphilic helix forms the membrane-anchoring domain of the Escherichia coli penicillinbinding protein 5. Mol. Microbiol. 1987, 1, 23-28. 
(56) Rabe, M.; Zope, H. R.; Kros, A. Interplay between lipid interaction and homo-coiling of membrane-tethered coiled-coil peptides. Langmuir 2015, 31, 9953-9964.

(57) Faivre, D.; Böttger, L. H.; Matzanke, B. F.; Schüler, D. Intracellular magnetite biomineralization in bacteria proceeds by a distinct pathway involving membrane-bound ferritin and an iron (II) species. Angew. Chem., Int. Ed. 2007, 46, 8495-8499.

(58) Weiner, S.; Addadi, L. Acidic macromolecules of mineralized tissues: the controllers of crystal formation. Trends Biochem. Sci. 1991, $16,252-256$.

(59) Grant, C. R.; Rahn-Lee, L.; LeGault, K. N.; Komeili, A. Genome Editing Method for the Anaerobic Magnetotactic Bacterium Desulfovibrio magneticus RS-1. Appl. Environ. Microbiol. 2018, 84, e01724e01718.

(60) Boucher, M.; Geffroy, F.; Prévéral, S.; Bellanger, L.; Selingue, E.; Adryanczyk-Perrier, G.; Péan, M.; Lefèvre, C. T.; Pignol, D.; Ginet, N.; Mériaux, S. Genetically tailored magnetosomes used as MRI probe for molecular imaging of brain tumor. Biomaterials 2017, 121, 167-178.

(61) Schwaminger, S. P.; Fraga-García, P.; Blank-Shim, S. A.; Straub, T.; Haslbeck, M.; Muraca, F.; Dawson, K. A.; Berensmeier, S. Magnetic One-Step Purification of His-Tagged Protein by Bare Iron Oxide Nanoparticles. ACS Omega 2019, 4, 3790-3799.

(62) Li, Q.; Barrett, D. G.; Messersmith, P. B.; Holten-Andersen, N. Controlling hydrogel mechanics via bio-inspired polymer-nanoparticle bond dynamics. ACS Nano 2016, 10, 1317-1324. 